TITLE:

\title{
The Effect of the Liquid-Crystalline Order on Releasing Dye Molecules from Liquid-Crystalline Nano- Emulsions
}

\section{$\operatorname{AUTHOR(S):~}$}

Bono, Shinji; Yokoyama, Masayuki; Takanishi, Yoichi; Yamamoto, Jun

\section{CITATION:}

Bono, Shinji ... [et al]. The Effect of the Liquid-Crystalline Order on Releasing Dye Molecules from Liquid-Crystalline Nano-Emulsions. Molecular Crystals and Liquid Crystals 2015, 610(1): 157-162

\section{ISSUE DATE:}

2015-07-06

URL:

http://hdl.handle.net/2433/201595

\section{RIGHT:}

This is an Accepted Manuscript of an article published by Taylor \& Francis in Molecular Crystals and Liquid Crystals on 2015, available online: http://www.tandfonline.com/10.1080/15421406.2015.1025625.; The full-text file will be made open to the public on 06 July 2016 in accordance with publisher's 'Terms and Conditions for Self-Archiving'.; This is not the published version. Please cite only the published version.; この論文は出版社版でありません。引用の際には出版社 版をご確認ご利用ください。 


\title{
The effect of the liquid-crystalline order on releasing dye molecules from liquid-crystalline nano-emulsions
}

\author{
Shinji Bono, ${ }^{1 *}$ Masayuki Yokoyama, ${ }^{2}$ Yoichi Takanishi ${ }^{1}$ and Jun Yamamoto ${ }^{1}$ \\ 1 Department of Physics, Graduate School of Science, Kyoto University, Kyoto, Japan \\ 2 Faculty of Medicine, The Jikei University School of Medicine, Tokyo, Japan
}

\begin{abstract}
We fabricated dye-containing liquid-crystalline nano-emulsions (LCNEs) dispersed in water, and studied the controllability of releasing dye molecules from the core of LCNEs into water. We estimated the concentration of the dye molecules held in the core of LCNEs from transmission spectrum. As a result, it was revealed that the dye molecules are released from the core of LCNEs below the characteristic release-temperature on cooling. Moreover, the depolarized static light scattering measurement was performed to estimate the Isotropic-Nematic (I-N) phase transition temperature of low molecular weight liquid crystals in the core of LCNEs. By controlling mixing ratio of binary liquid crystalline mixture, we tuned the I-N phase transition temperature. The releasetemperature completely corresponds to the I-N phase transition temperature in the core of LCNEs within the whole mixing ratio. Thus, we confirmed that the emergence of the liquid-crystalline order in the core of LCNEs plays a principal role in releasing the dye molecules from LCNEs.
\end{abstract}

\section{Introduction}

The purposes of drug delivery system (DDS) are to hold drugs in carriers and to deliver them to the diseased part in vivo [1-2]. Since the stimuli-responsive materials can control releasing loaded drugs by means of external stimuli, they enable us to selectively release drugs [3-5]. Previously, we have succeeded in dispersing the liquid-crystalline nano-emulsions (LCNEs) with diameter of a few hundred nanometers. In such a LCNE system, the shell and the core of LCNEs are occupied by surfactants and nematic low molecular weight liquid crystals (LMWLCs), respectively [6]. The liquid-crystalline (LC) order in the core of LCNEs abruptly emerges at the IsotropicNematic (I-N) phase transition. Solubility of solute molecules in LMWLCs also greatly decreases at the I-N phase transition, because the order parameter of LMWLCs couples with the concentration of solute molecules in LMWLCs [7]. Thus, LCNEs can push out the solute molecules from the core of LCNEs into water triggered by the I-N phase transition in the core of LCNEs. Therefore, LCNEs are expected to work as temperature-responsive carriers for DDS.

In this paper, we adopted a dye as a cargo-molecule, whose absorbance spectrum in LMWLCs differs from that in water, to the water solution of LCNEs [8], and measured temperature dependence of absorbance to estimate concentration of the dye molecules held in the core of LCNEs. To separate the effect of the LC order on releasing the dye molecules from other temperature effects, e.g. solubility [9] and external osmotic pressure [10], we made LCNEs constituted by the binary mixture LMWLCs whose I-N phase transition temperature controlled by their mixing ratio [11]. We confirmed that the dye molecules are successfully released from the core of LCNEs, and that releasing is triggered by the emergence of the LC order in the core of LCNEs.

\section{Experimental}

We used the binary $\mathrm{LC}$ mixture of $5 \mathrm{CB}\left(\mathrm{N}-35^{\circ} \mathrm{C}-\mathrm{Iso}\right)$ and $7 \mathrm{CB}\left(\mathrm{N}-42^{\circ} \mathrm{C}-\mathrm{Iso}\right)$. Mixing ratio $\phi$ is defined as $w_{7} /\left(w_{5}+w_{7}\right)$, where $w_{5}$ and $w_{7}$ are weight of $5 \mathrm{CB}$ and $7 \mathrm{CB}$, respectively. We evaluated the phase transition temperatures of the binary LC mixtures in the bulk state by measuring temperature dependence of retardation of each binary LC mixture. We got the water solutions of LCNEs in the following way. An amphiphilic diblock copolymer, Poly (ethylene oxicide-b-6-[4'cyanobiphenyl-4-yloxy]hexyl methacrylate), whose side chain contains mesogenic part and the binary LC mixture are mixed with the constant weight ratio in acetone. After removing acetone by evaporation, we added water into this mixture and sonicated it for more than two hours at $60{ }^{\circ} \mathrm{C}$. We purified this solution with a syringe filter whose diameter is $1 \mu \mathrm{m}$. As a cargo-molecule, Nile Blue A perchlorate was used [8]. By adding the water solution of Nile Blue A perchlorate into the water 
solution of LCNEs, we got dye-containing LCNEs. Concentration of the binary LC mixture and the dye molecules of LCNEs are about $0.1 \mathrm{wt} \%$ and about $4 \times 10^{-3} \mathrm{wt} \%$, respectively.

LCNEs are uniformly dispersed in water and freely rotate because of thermally excited Brownian motions. Hence, the macroscopic optical anisotropy completely disappears, even if each micelle has the anisotropy caused by the LC order in the core of LCNEs. To identify the existence of the anisotropy in the core of LCNEs, the light scattering technique was used. We measured temperature dependence of the $\mathrm{VH}$ static light scattering intensities $\left(I_{\mathrm{VH}}\right)$ of LCNEs. $I_{\mathrm{VH}}$ reflects the anisotropic order of the binary LC mixtures in the core of LCNEs [6]. According to the theory of light scattering from a nematic droplet in isotropic solvent [12], $I_{\mathrm{VH}}$ is quadratically proportional to the dielectric anisotropy of LCNEs, $\left(\varepsilon_{\|}-\varepsilon_{\perp}\right)$, where $\varepsilon_{\|}$and $\varepsilon_{\perp}$ are the components of dielectric constants parallel and perpendicular to the anisotropic axis, respectively. Since the dielectric anisotropy should indicate the LC order, we can evaluate the I-N phase transition temperature of the binary LC mixtures confined in the core of LCNEs by measuring $I_{\mathrm{VH}}$.

\section{Results and Discussion}

Fig.1 shows temperature dependence of $I_{\mathrm{VH}}$ of LCNEs with various values of mixing ratio $\phi . I_{\mathrm{VH}}$ was normalized so that maximum and minimum values of $I_{\mathrm{VH}}$ become one and zero, respectively. Irrespective of $\phi, I_{\mathrm{VH}}$ increases with decreasing temperature. For LCNEs whose core is composed of pure $7 \mathrm{CB}(\phi=1), I_{\mathrm{VH}}$ abruptly increases around $39.5^{\circ} \mathrm{C}$ on cooling. Since the temperature of abrupt change in $I_{\mathrm{VH}}$ was close to the I-N phase transition temperature of $7 \mathrm{CB}$ in the bulk state $\left(\sim 42^{\circ} \mathrm{C}\right)$, the result is originated from the emergence of the finite LC order due to the I-N phase transition in the core of LCNEs [6]. We defined the phase transition temperature as $T_{\mathrm{c}}^{\mathrm{NE}}$. This abrupt change reflecting the I-N phase transition in the core of LCNEs was observed for all $\phi s$. It is evident that $T_{\mathrm{c}}^{\mathrm{NE}}$ becomes lower with the decrease in $\phi$, and is always close to the phase transition temperature of the binary LC mixture with the same $\phi$ in the bulk state. Hence, we found that $T_{\mathrm{c}}^{\mathrm{NE}}$ can be controlled by changing $\phi$, even if the binary LC mixture is confined in the core of LCNEs.

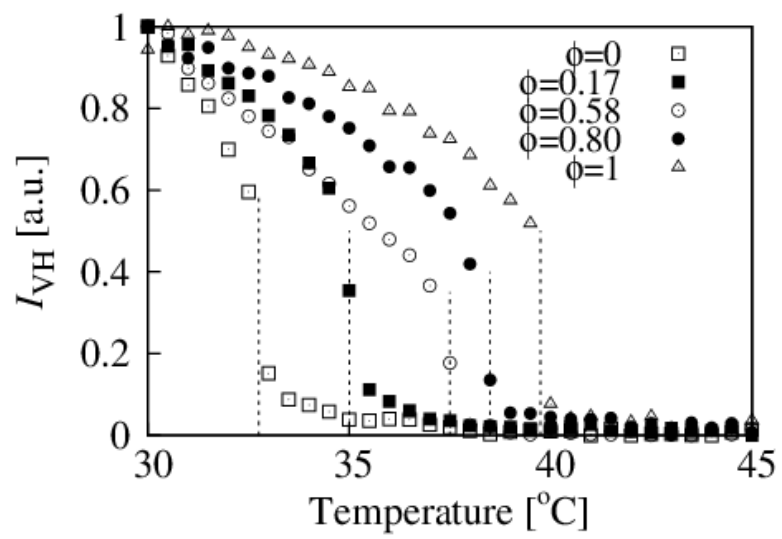

Fig.1 Temperature dependence of $I_{\mathrm{VH}}$ of LCNEs with various values of $\phi$.

In order to identify whether the dye molecules are held in the core of LCNEs or released into water, we measured transmission spectra of three kinds of samples; dye-containing LCNEs with $\phi=0.58$ (sample 1), the water solution of the dye molecules (sample 2), and the LMWLC (7CB) doped with the dye molecules (sample 3). We got normalized transmission spectra of the dyecontaining LCNE by normalizing spectra of sample 1 by those of LCNEs without dye molecules, because transmittance decreases due to the scattering of LCNEs themselves. For sample 2 and sample 3, we normalized the maximum value with $100 \%$. Comparing these normalized spectra, we examined whether the dye molecules are dissolved in water or in the core of LCNEs. The normalized transmission spectra of sample 1 at $60{ }^{\circ} \mathrm{C}$ and $30{ }^{\circ} \mathrm{C}$ are shown in Fig. 2. We also superimposed transmission spectra of sample 2 and sample 3. The dye molecules dissolved in LMWLCs absorb visible light around $525 \mathrm{~nm}$ in addition to around $640 \mathrm{~nm}$ (sample 3). Transmission spectrum of sample 1 at $60{ }^{\circ} \mathrm{C}$ also has dips due to the absorption of dye molecules around $525 \mathrm{~nm}$ 
(indicated by a black arrow) and 640nm (indicated by a broken black allow) as similar to the spectrum of dye molecules in LMWLCs (sample 3). Thus, it is confirmed that the dye molecules are held in the core of LCNEs. On the other hands, at $30{ }^{\circ} \mathrm{C}$ the dip around $525 \mathrm{~nm}$ disappears, but appears around $600 \mathrm{~nm}$ (indicated by a dotted gray arrow), as similar to the transmission spectrum of the dye molecules dissolved in water (sample 2). Finally, it can be concluded that the dye molecules held in the core of LCNEs at $60^{\circ} \mathrm{C}$ are released into water at $30^{\circ} \mathrm{C}$ from LCNEs.

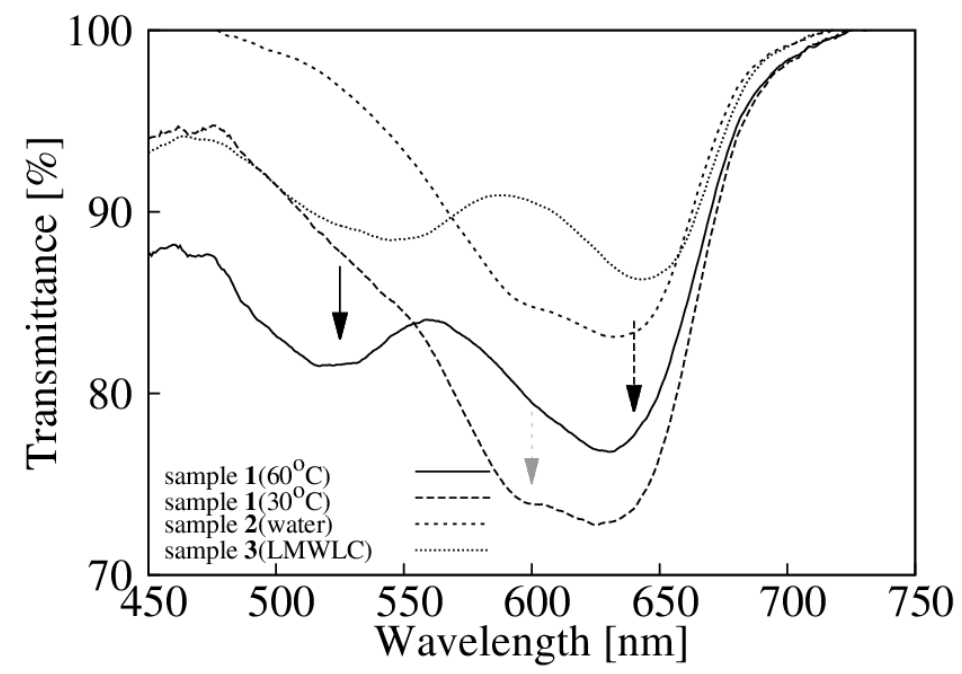

Fig.2 The transmission spectra of the dye-containing LCNE with $\phi=0.58$ (sample 1) at $60{ }^{\circ} \mathrm{C}$ and 30 ${ }^{\circ} \mathrm{C}$. We also superimposed transmission spectra of the dye molecules dissolved in water and in the LMWLCs. The arrows are placed at $525 \mathrm{~nm}$ (black) and $600 \mathrm{~nm}$ (dotted gray), $640 \mathrm{~nm}$ (broken black).

We estimated the concentration of the dye molecules held in the core of LCNEs, $C_{\text {hold }}$, from transmittance at $525 \mathrm{~nm}, \tau_{\lambda=525 \mathrm{~nm}}$. According to the Beer-Lambert law [13], $\tau_{\lambda=525 \mathrm{~nm}}$ is related to $C_{\text {hold }}$ as follows,

$$
-\frac{\log _{10}\left(I_{\lambda=525 \mathrm{~nm}} / I_{0}\right)}{C_{\mathrm{LC}} L}=\alpha \frac{C_{\mathrm{dye}}}{100} C_{\text {hold }}
$$

where $L, C_{\mathrm{LC}}$ and $C_{\mathrm{dye}}$ are thickness of sample $(\sim 1 \mathrm{~mm})$, the concentration of the binary LC mixture and that of the dye molecules, respectively. $\alpha\left(\sim 6.0 \times 10^{-3}\left[\mu \mathrm{m}^{-1}\right]\right)$ is the absorption coefficient of the dye molecules, determined from the transmission measurement of sample 3. Fig. 3 shows temperature dependence of $C_{\text {hold }}$ for sample 1 . We can define the release-temperature, $T_{\mathrm{r}}^{\text {dye }}$, where $C_{\text {hold }}$ is abruptly changed. Above $T_{\mathrm{r}}^{\text {dye }}$, about $40 \%$ of the dye molecules are held in the core of LCNEs. At $T-T_{\mathrm{r}}^{\text {dye }} \sim-5{ }^{\circ} \mathrm{C}$, about $30 \%$ of dye molecules are released into water and only $10 \%$ of the dye molecules remain in the core of LCNEs. 


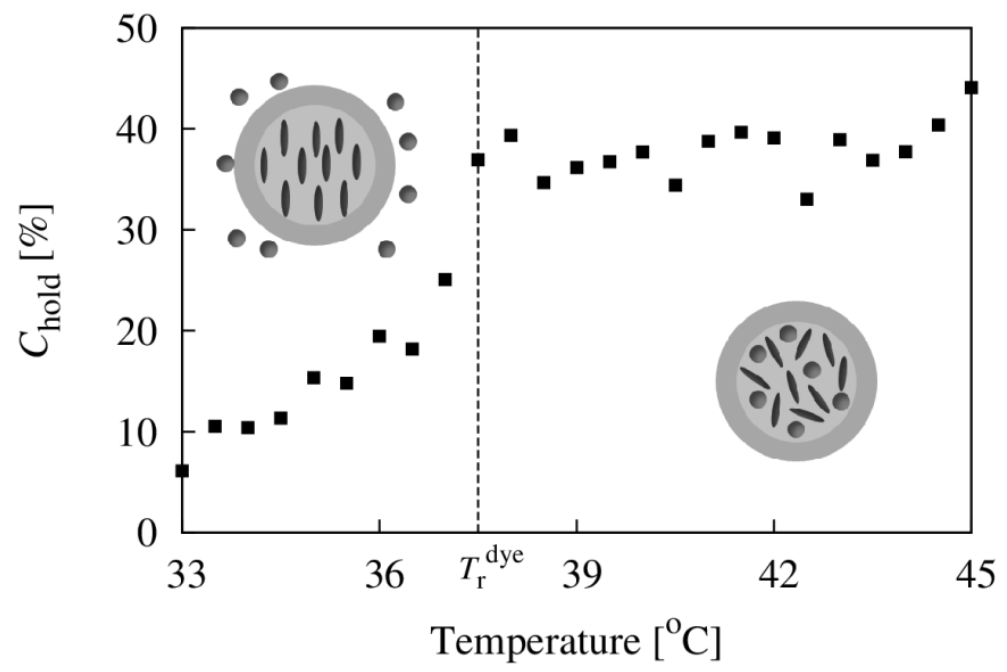

Fig.3 Temperature dependence of the $C_{\text {hold }}$ for sample 1 . The broken line shows the releasetemperature.

Fig. 4 shows $\phi$ dependence of the release temperature, $T_{\mathrm{r}}^{\text {dye }}$ and the I-N phase transition temperature of the core of LCNEs, $T_{\mathrm{c}}^{\mathrm{NE}}$, associated with the I-N phase transition temperature of the binary LC mixture in the bulk state, $T_{\mathrm{c}}^{\text {bulk }} . T_{\mathrm{c}}^{\mathrm{NE}}$ and $T_{\mathrm{r}}^{\text {dye }}$ increase with the increase in $\phi$. Moreover, they are always equivalent to each other within the whole mixing ratio. This result suggests that releasing dye molecules from the core of LCNEs can be controlled by the change of the LC order in the core, and that emergence of the LC order can push out dye molecules into water. On the contrary, the dye molecules are stably held in the core, if the binary LC mixture in the core of LCNEs is isotropic. Furthermore, $T_{\mathrm{c}}^{\mathrm{NE}}$ is slightly lower than $T_{\mathrm{c}}^{\text {bulk}}$, which originates from impurity and confined effects as follows. First, hydrophobic chains of surfactants on the surface of LCNEs work as impurities, because they partially mix with the binary LC mixture in the core of LCNEs. Generally speaking, impurities, such as polymers, weaken the LC order parameter, and hence reduce the I-N phase transition temperature [7]. On the other hand, when the uniaxial nematic phase is confined in the spherical nano-emulsions, director needs deformation to match the shape of the inner surface of the container (surfactant). Additionally, defects are introduced under the topological constraint on the director distortion. Both require the penalty of the excess free energy, which causes reduction of the I-N phase transition temperature $[6,14]$.

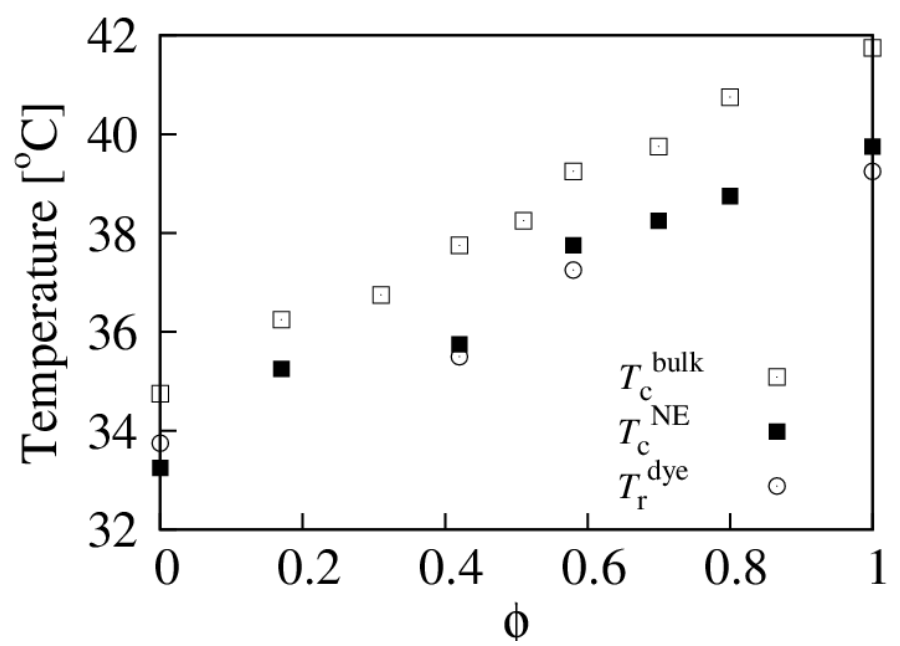

Fig. $4 \phi$ dependence of the I-N phase transition temperature of the binary LC mixture in the bulk state $T_{\mathrm{c}}^{\text {bulk }}$, and that in the core of LCNEs $T_{\mathrm{c}}^{\mathrm{NE}}$, and $T_{\mathrm{r}}^{\text {dye }}$. 


\section{Conclusion}

In conclusion, we revealed that the I-N phase transition can trigger off release of the dye molecules from the core of LCNEs into water, and that LCNEs can work as carriers. The static light scattering measurements give us the correct information about the I-N phase transition of the binary LC mixture in the core of LCNEs, whereas the LCNEs are macroscopically transparent and isotropic. The concentration ratio of the dye molecules held in the core to those released into water was estimated from the transmission spectra of the dye-containing LCNEs. Moreover, we compared I-N phase transition temperature in the core of LCNEs and the release-temperature of the dye molecules, and confirmed that the change of LC order plays a principal role in releasing the dye molecules.

\section{Acknowledgements}

This work was partially supported by a JSPS Core-to-Core program "Non-equilibrium dynamics of soft matter and information", and Grant-in-Aid for JSPS Fellows Grant Number 13J01166, and Grants-in-Aid for Scientific Research.

\section{References}

[1] Iyer, A. K., Khaled, G., Fang J., \& Maeda, H. (2006). Drug Discov. Today, 11, 812.

[2] Rösler, A., Vandermeulen, G. W. M., \& Klok, H. A. (2001). Adv. Drug. Deliv. Rev., 53, 95.

[3] Nishihara, M., Murakami, Y., Shinoda, T., Yamamoto, J., \& Yokoyama, M. (2008). Chem. Lett. 37, 1214.

[4] Zhang, Z. X., Qiu, L. Y., \& Zhu, Y. J. (2006). J. Biomed. Mater. Res. A. 76, 773.

[5] Wei, H., Zhang, X., Cheng, C., Cheng, S. X., \& Zhuo, R. X. (2007). Biomaterials. $28,99$.

[6] Bono, S., Takanishi, Y., \& Yamamoto, J. in preparation.

[7] Samitsu, S., Takanishi, Y., \& Yamamoto, J. (2010) Nat. Mater. 9, 819.

[8] Jose, J., Ueno, Y., \& Burgess, K. (2009) Chem. Eur. J. 15, 418.

[9] Muschol, M., \& Rosenberger, F. (1997) J. Phys. Chem. 107, 1953.

[10] Petsev, D. N., Wu, X., Galkin, O., \& Vekilov, P. G. (2003) J. Phys. Chem. B. 107, 3921.

[11] Demus, D., Fietkau, Ch., Shubert, R. \& Kehlen, H. (1974) Mol. Cryst. Liq. Cryst. 25, 215.

[12] Zumer, S., \& Doane, J. W. (1986) Phys. Rev. A, 34, 3373.

[13] Boas, D. A., Gaudette, T., Strangman, G., Cheng, X., Marota, J. J. A., \& Mandeville, B. (2001) NeuroImage 13, 76.

[14] Kutnjak, Z., Kralj. S., Lahajnar, G., \& Zumer, S. (2003) Phys. Rev. E, 68, 021705. 